OPEN ACCESS

Edited by:

Suhelen Egan,

University of New South Wales,

Australia

Reviewed by:

Kyung-Woo Lee,

Konkuk University, South Korea

Suzanne Lynn Ishaq,

Montana State University,

United States

*Correspondence:

Yong Zhao

yzhao818@hotmail.com

Hongfu Zhang

zhanghongfu@caas.cn

tThese authors are co-first author.

Specialty section: This article was submitted to

Microbial Symbioses,

a section of the journal

Frontiers in Microbiology

Received: 27 March 2017 Accepted: 17 May 2017

Published: 02 June 2017

Citation:

Feng Y, Min L, Zhang W, Liu J, Hou Z, Chu M, Li L, Shen W, Zhao $Y$ and Zhang $H$ (2017) Zinc

Oxide Nanoparticles Influence Microflora in lleal Digesta

and Correlate Well with Blood

Metabolites. Front. Microbiol. 8:992.

doi: 10.3389/fmicb.2017.00992

\section{Zinc Oxide Nanoparticles Influence Microflora in lleal Digesta and Correlate Well with Blood Metabolites}

Yanni Feng ${ }^{1 \dagger}$, Lingjiang Min ${ }^{1+}$, Weidong Zhang1, Jing Liu' ${ }^{2}$, Zhumei Hou ${ }^{3}$, Meiqiang Chu ${ }^{1}$, Lan Li $^{1}$, Wei Shen ${ }^{1}$, Yong Zhao ${ }^{1,4 *}$ and Hongfu Zhang ${ }^{4 *}$

${ }^{1}$ College of Animal Science and Technology, Qingdao Agricultural University, Qingdao, China, ${ }^{2}$ Core Laboratories of Qingdao Agricultural University, Qingdao, China, ${ }^{3}$ College of Marine Science and Technology, Qingdao Agricultural University, Qingdao, China, ${ }^{4}$ State Key Laboratory of Animal Nutrition, Institute of Animal Sciences, Chinese Academy of Agricultural Sciences, Beijing, China

Zinc oxide nanoparticles (ZnO NPs) are used widely in consumer and industrial products, however, their influence on gut microbiota and metabolism and their mutual interactions are not fully understood. In this study, the effects of ZnO NPs on ileal bacterial communities, plasma metabolites, and correlations between them were investigated. Hens were fed with different concentrations of ZnO NPs [based on Zn; 0 mg/kg (control), $25 \mathrm{mg} / \mathrm{kg}, 50 \mathrm{mg} / \mathrm{kg}$, and $100 \mathrm{mg} / \mathrm{kg}$ ] for 9 weeks. Subsequently, ileal digesta and blood plasma were collected for analysis of microflora and metabolites, respectively. The V3-V4 region of the 16S rRNA gene of ileal digesta microbiota was sequenced using the Illumina HiSeq 2500 platform. The predominant bacterial community in the ileum belongs to the phylum Firmicutes. The richness of the bacterial community was negatively correlated with increasing amounts of ZnO NPs $(r=-0.636, P<0.01)$; when ZnO NP levels were at $100 \mathrm{mg} / \mathrm{kg}$, microbiota diversity was significantly decreased $(P<0.05)$. The community structure determined by LEfSe analysis indicated that Bacilli, Fusobacteria, and Proteobacteria were changed, and Lactobacillus was reduced by ZnO NPs. Moreover, metabolism as analyzed by nuclear magnetic resonance (NMR) indicated that glucose, some amino acids, and other metabolites were changed by ZnO NPs. Choline, lactate, and methionine were positively correlated with bacterial richness. In summary, ZnO NPs could influence the levels of microflora in ileal digesta, particularly Lactobacillus. Furthermore, the richness of the microbiota was related to changes in choline, lactate, and methionine metabolism.

Keywords: $\mathrm{ZnO}$ nanoparticles, microflora, ileal digesta, metabolism, correlation

\section{INTRODUCTION}

Zinc oxide nanoparticles ( $\mathrm{ZnO} \mathrm{NPs}$ ) are a new source of zinc with a diameter of $<100 \mathrm{~nm}$; they are widely used in consumer and industrial products. They offer excellent properties for antibacterial action, treatment of allergic diseases, and anti-cancer drug delivery (Kim et al., 2014; Patel et al., 2016). It is reported that ZnO NPs can effectively inhibit pathogenic yeast and Candida albicans (Lipovsky et al., 2011), with improved antibacterial action over copper oxide nanoparticles 
(CuO NPs) and ferric oxide nanoparticles $\left(\mathrm{Fe}_{2} \mathrm{O}_{3}\right.$ NPs; Azam et al., 2012). Silva and Girard (2016) also discovered that $\mathrm{ZnO}$ NPs could prevent human eosinophil apoptosis through inhibiting caspase- 1 and caspase-4. The adverse effects of $\mathrm{ZnO}$ NPs on organisms have been investigated under many conditions and the toxic effects are highly related to NP size and shape (Saptarshi et al., 2015). The augmentation of reactive oxygen species (ROS) and $\mathrm{Zn}^{2+}$ are the greatest potential causes of $\mathrm{ZnO}$ NP cytotoxicity (Wang et al., 2014). Furthermore, ZnO NPs have some influence on metabolism as evidenced by metabolites in mouse urine (Yan et al., 2012) and disturbed glucose metabolism in lung epithelial cells (Lai et al., 2015).

The bacterial community plays an important role in nutrition, immune system development, metabolic homeostasis, and antipathogenic action for humans and animals (Shin et al., 2011; Pan and Yu, 2014). Many factors can affect gut microflora, including the host, different diseases, diets, and additives, etc. It has been shown that fructose dianhydride-enriched caramel (FC) and the garlic derivative propyl propane thiosulfonate (PTS-O) influence the composition of ileal mucosa-associated microbiota (Ruiz et al., 2015), while phytogenic feed additives could increase Lactobacillus levels in the ceca of broiler chickens (Murugesan et al., 2015). Furthermore, the exogenous enzyme improves nutrient digestibility and reduces the number of E. coli (Rodriguez et al., 2012). Short-chain fatty acids and monoglycerides inhibit the contamination of Campylobacter jejuni in broiler chickens (Guyard-Nicodeme et al., 2016). Reed et al. (2015) proved that the richness and diversity of cecum microbiota were altered in chickens with chronic dietary $\mathrm{Zn}$ deficiency. Shao et al. (2014) found that zinc regulated the cecal microbial community by increasing the number of total bacteria and beneficial Lactobacillus bacteria, and reducing the number of Salmonella in broilers. The antibiotic zinc bacitracin induced specific changes in the cecal microbiota of broiler chicken (Gong et al., 2008; Torok et al., 2011; Costa et al., 2017; Crisol-Martinez et al., 2017). ZnO NPs produced attractive antibacterial properties because of the increased specific surface area as the reduced particle size with enhanced particle surface reactivity (Sirelkhatim et al., 2015). Hu et al. (2017) has found that the microbial species richness in the EBPR system was reduced by $\mathrm{ZnO} \mathrm{NPs}$ and higher concentration $\mathrm{ZnO}$ NPs induced more microbial community shift. ZnO NPs-caused antibacterial effects may be due to a combination of $\mathrm{Zn}^{2+}$ ions and induction of ROS formation (Frohlich and Frohlich, 2016).

Although there are still few reports on the possible toxicological effects of nanoparticles on microbiota/microbiome, and on their possible clinical consequences, available data suggest that carbon nanotubes (CNTs), TiO2 NPs, CeO2 NPs, ZnO $\mathrm{NPs}, \mathrm{SiO}_{2} \mathrm{NPs}$, and $\mathrm{Ag}$ NPs may affect the microbiota in intestine (Pietroiusti et al., 2016). Ag NPs and $\mathrm{Ti}_{2} \mathrm{O}$ NPs caused changes in the microbiota and mucus in intestine (Mercier-Bonin et al., 2016). Taylor et al. (2015) used an in vitro colon model found that $\mathrm{ZnO}$ NPs caused significant changes to the microbial community's phenotype, which might be related to overall health effects. Moreover, it has been shown that ZnO NPs can affect soil microflora and the growth of dominant bacteria, namely Bacillus subtilis and Pseudomonas aeruginosa (Ge et al., 2011; Santimano and Kowshik, 2013). However, the effects of ZnO NPs on gut microflora of domestic animals and on the relationship between metabolites and gut microflora are not yet understood. Since gut microflora are of great importance to metabolism, the objective of this investigation was to explore the influence of $\mathrm{ZnO}$ NPs on the bacterial community of hen ileal digesta, the metabolites in the plasma, and the relation between them.

\section{MATERIALS AND METHODS}

\section{The Characterization of $\mathrm{ZnO}$ Nanoparticles}

Zinc oxide nanoparticles were synthesized by Beijing DK Nano Technology Co. LTD (Beijing, China) as reported in our recent publications (Supplementary Figure S1) (Zhao et al., 2016). The characteristics of $\mathrm{ZnO}$ NPs (morphology, size, agglomeration, etc.) were determined by transmission electron microscopy (TEM; JEM-2100F, JEOL Inc., Japan) and dynamic light scattering (DLS) particle size analyzer (Nano-Zetasizer-HT, Malvern Instruments, United Kingdom).

\section{Animal Husbandry and Sample Collection}

All animal experimental procedures were followed according to the regulations of the animal ethics committee of the Qingdao Agricultural University. All Jinghong-1 hens used in the experiment were housed in a commercial poultry building at Maochangyuan Co. (Qiangdao, China). Diets and nutrients are shown in Supplementary Table S1. Briefly, 160 six-week-old hens were equally divided into four different treatments: control (C), no zinc addition; ZnO-NP-25 (25 mg/kg ZnO NPs; based on the concentration of zinc); ZnO-NP-50 (50 mg/kg ZnO NPs); and $\mathrm{ZnO}-\mathrm{NP}-100$ (100 mg/kg ZnO NPs) with four repeats in each treatment (10 hens/repeat). At 15 weeks, two hens from each repeat were euthanized after recording body weight and collection of blood samples. Ileal digesta (eight samples of each group) was packaged and immersed in liquid nitrogen before being stored at $-80^{\circ} \mathrm{C}$ for DNA isolation. Blood samples (eight samples of each group) were centrifuged at $3000 \mathrm{rpm}$ for $15 \mathrm{~min}$ at $4^{\circ} \mathrm{C}$ and the plasma was stored at $-80^{\circ} \mathrm{C}$ for metabolite detection.

\section{The Sequencing of Microbiota from lleal Digesta Samples DNA Extraction}

Total genomic DNA of ileal digesta was extracted using an E.Z.N.A. ${ }^{\circledR}$ Stool DNA Kit (Omega Bio-tek Inc., United States) according to the manufacturer's introduction. DNA quantity and quality were monitored with NanoDrop 2000 (Thermo Scientific, United States) and 1\% agarose gel. Totally 32 ileal digesta DNA samples were extracted and the two ileal digesta DNA from the same replicate were equally mixed and 16 samples were applied for sequencing $(n=16)$. 


\section{Library Preparation and Sequencing}

The V3-V4 region of the 16S rRNA gene was amplified using the primers MPRK341F (5'-CCTAYGGGRBGCASCAG-3') and MPRK806R (5'-GGACTACNNGGGTATC TAAT-3') (Yu et al., 2005) with Barcode. The volume of PCR reactions was $30 \mu \mathrm{L}$ with $15 \mu \mathrm{L}$ Phusion ${ }^{\circledR}$ High-Fidelity PCR Master Mix (New England Biolabs), $0.2 \mathrm{Mm}$ primers and $10 \mathrm{ng}$ DNA. The thermal cycle was carried out with initial denaturation at $h$, followed by 30 cycles of $98^{\circ} \mathrm{C}$ for $10 \mathrm{~s}, 50^{\circ} \mathrm{C}$ for $30 \mathrm{~s}$ and $72^{\circ} \mathrm{C}$ for $30 \mathrm{~s}$, and a final extension at $72^{\circ} \mathrm{C}$ for $5 \mathrm{~min}$. PCR products were mixed in equidensity ratios and purified using a GeneJET Gel Extraction Kit (Thermo Scientific, United States). The sequencing libraries were generated with NEB Next ${ }^{\circledR}$ Ultra $^{\text {TM }}$ DNA Library Prep Kit for Illumina (NEB, United States) following the manufacturer's recommendations and index codes were added. Lastly, the library was sequenced on the Illumina HiSeq 2500 platform and 250 bp paired-end reads were generated at the Novo gene. The paired-end reads were merged by using FLASH $\left(\mathrm{V} 1.2 .7^{1}\right)$. The quality of the Tags was controlled in QIIME (V1.7.02) as according to the protocol described by Caporaso et al., 2010), meanwhile all chimeras were removed (Edgar et al., 2011; Haas et al., 2011). The "Core Set" of the Greengenes database ${ }^{3}$ was applied for classification, and sequences with $\geq 97 \%$ similarity were assigned to the same operational taxonomic units (OTUs) (Magoc and Salzberg, 2011).

\section{Analysis of Sequencing Data}

Operational taxonomic unit abundance information was normalized using a standard of sequence number corresponding to the sample with the least sequences. The alpha diversity index was calculated with QIIME (Version 1.7.0). The Unifrac distance was obtained by QIIME (Version 1.7.0), and PCoA (principal coordinate analysis) was performed using $\mathrm{R}$ software (Version 2.15.3). The Linear discriminate analysis effect size (LEfSe) was performed to determine differences in abundance, the threshold of LDA score was 4.0. GraphPad Prism7 software was used to produce the graphs.

\section{Analysis of Metabolites in Blood Plasma Blood Plasma Preparation and Nuclear Magnetic Resonance (NMR) Spectrum Processing}

The detection of metabolites in hen plasma followed the method reported by Wan et al. (2016). In total, $600 \mu \mathrm{L}$ of solution (including $200 \mu \mathrm{L}$ plasma, $80 \mu \mathrm{L} \mathrm{D} \mathrm{D}_{2} \mathrm{O}$ and $\mathrm{NaCl}$ media to a final concentration of $0.9 \% \mathrm{NaCl}$ ) was transferred to a $5 \mathrm{~mm}$ nuclear magnetic resonance (NMR) tube. A Bruker Avance III $600 \mathrm{MHz}$ NMR spectrometer equipped with an inverse detection cryoprobe (Bruker Biospin, Rheinstetten, Germany) was used to obtain the ${ }^{1} \mathrm{H}$ NMR spectra for plasma samples.

\footnotetext{
${ }^{1}$ http://ccb.jhu.edu/software/FLASH/

${ }^{2}$ http://qiime.org/index.html

${ }^{3}$ http://greengenes.lbl.gov/cgi-bin/nph-index.cgi
}

\section{Data Analysis of the ${ }^{1} \mathrm{H}$ NMR Spectra}

The plasma spectra were corrected with phase and baseline distortions, the spectra were then referenced to $\mathrm{TSPd}_{4}$ $(\delta 0.000)$ and plasma $(\delta 0.5-8.5)$. Imperfect water suppression $(\delta 4.40-5.20)$ was excluded. The obtained spectra were normalized to the total sum of intensity and the data were analyzed with SIMCA-P software (version 11.0, Umetrics, Umea, Sweden). All Tukey's multiple comparison tests using one-way ANOVA were performed with GraphPad Prism7. Correlation analysis was performed using SPSS version 22.

\section{RESULTS}

\section{Effects on Body Weight}

After 9 weeks of treatment, individual hen body weights were $0.92-1.48 \mathrm{~kg}$, and these remained unaltered by treatments using different concentrations of $\mathrm{ZnO}$ NP supplement (Figure 1).

\section{Changes of Microflora in Ileal Digesta Patterns of Predominant Bacterial Communities in lleal Digesta}

Based on relative abundance, $\mathrm{ZnO}$ NPs altered the predominant bacteria in hen ileal digesta. In the control group, the predominant bacteria were Firmicutes, Clostridia and Bacilli, Clostridiale and Lactobacillales, Clostridiaceae and Lactobacillaceae, SMB53, and Lactobacillus; while Firmicutes, Clostridia, Clostridiale, Clostridiaceae, and SMB53 were the predominant bacteria in $\mathrm{ZnO} \mathrm{NP}$ treatments (Figure 2 and Table 1).

\section{Diversity of Bacterial Communities in Ileal Digesta}

In total, 815828 clean tags were obtained, with an average efficiency of $83.37 \%$. The rarefaction curve revealed that the sequence number was reliable for further analysis (Figure 3A), meanwhile, the observed species of ileal digesta microbiota

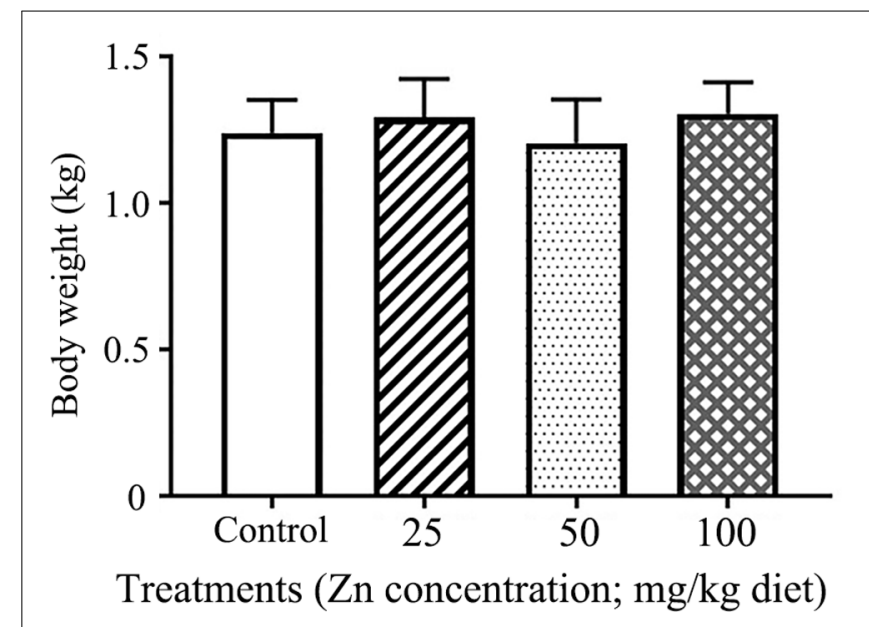

FIGURE 1 | Effects of ZnO NP treatment on hen body weight. The $Y$-axis represents body weight $(\mathrm{kg})$, and the $X$-axis represents the treatment group. 


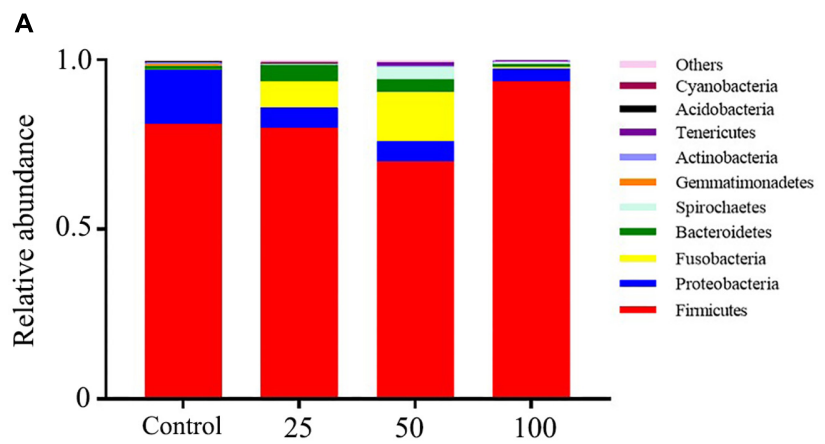

Treatments ( $\mathrm{Zn}$ concentration; $\mathrm{mg} / \mathrm{kg}$ diet)

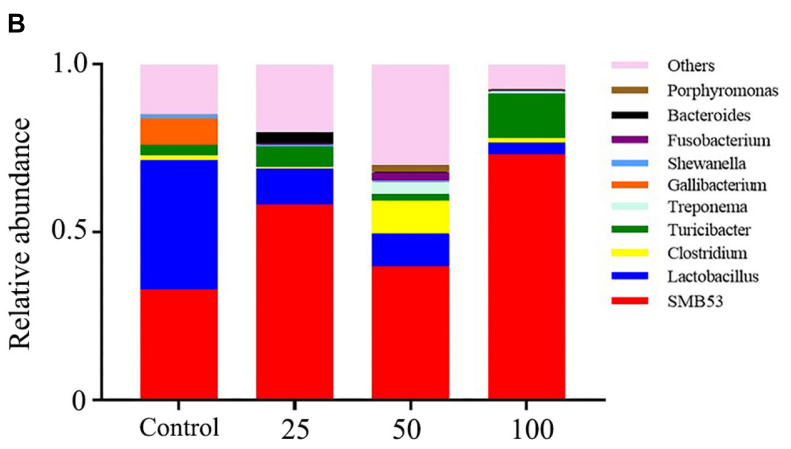

Treatments ( $\mathrm{Zn}$ concentration; $\mathrm{mg} / \mathrm{kg}$ diet)

FIGURE 2 | The relative richness of microbiota at phylum and genus level. The representative sequences for each OUT was used to annotate taxonomic information: (A) phylum, (B) genus.

TABLE 1 | The relative abundance of gut microflora in ileal digesta (\%).

\begin{tabular}{|c|c|c|c|c|c|}
\hline Taxonomy & Phylum & Class & Order & Family & Genus \\
\hline & Proteobacteria 15.94 & Bacilli 43.71 & Lactobacillales 39.94 & Lactobacillaceae 38.34 & Lactobacillus 38.33 \\
\hline & & & Others 5.47 & Others 10.63 & Others 14.89 \\
\hline \multirow[t]{3}{*}{$\mathrm{ZnO} 25$} & Firmicutes 79.89 & Clostridia 61.83 & Clostridiales 61.83 & Clostridiaceae 58.87 & SMB53 58.18 \\
\hline & Proteobacteria 5.97 & Bacilli 17.88 & Lactobacillales 11.53 & Lactobacillaceae 10.83 & Lactobacillus 10.83 \\
\hline & & & Turicibacterales 6.00 & Others 12.01 & Others 20.20 \\
\hline \multirow[t]{4}{*}{$\mathrm{ZnO50}$} & Firmicutes 70.07 & Clostridia 57.54 & Clostridiales 57.54 & Clostridiaceae 53.39 & SMB53 39.94 \\
\hline & Proteobacteria 5.91 & Bacilli 12.51 & Lactobacillales 10.27 & Lactobacillaceae 9.59 & Lactobacillus 9.59 \\
\hline & Fusobacteria 14.39 & Fusobacteriia 14.39 & Fusobacteriales 14.39 & Fusobacteriaceae 15.52 & Clostridium 9.79 \\
\hline & Bacteroidetes 3.94 & Bacteroidia 3.91 & Bacteroidales 3.91 & Spirochaetaceae 3.49 & Others 30.05 \\
\hline & Proteobacteria 3.81 & Bacilli 18.18 & Lactobacillales 4.44 & Lactobacillaceae 3.62 & Lactobacillus 3.57 \\
\hline & & & Turicibacterales 13.35 & Turicibacteraceae 13.35 & Turicibacter 13.35 \\
\hline & & & Others 3.66 & Others 6.00 & Others 7.38 \\
\hline
\end{tabular}

This table only documented the group unites taxa whose abundance (each) exceed $3.5 \%$ of the total.
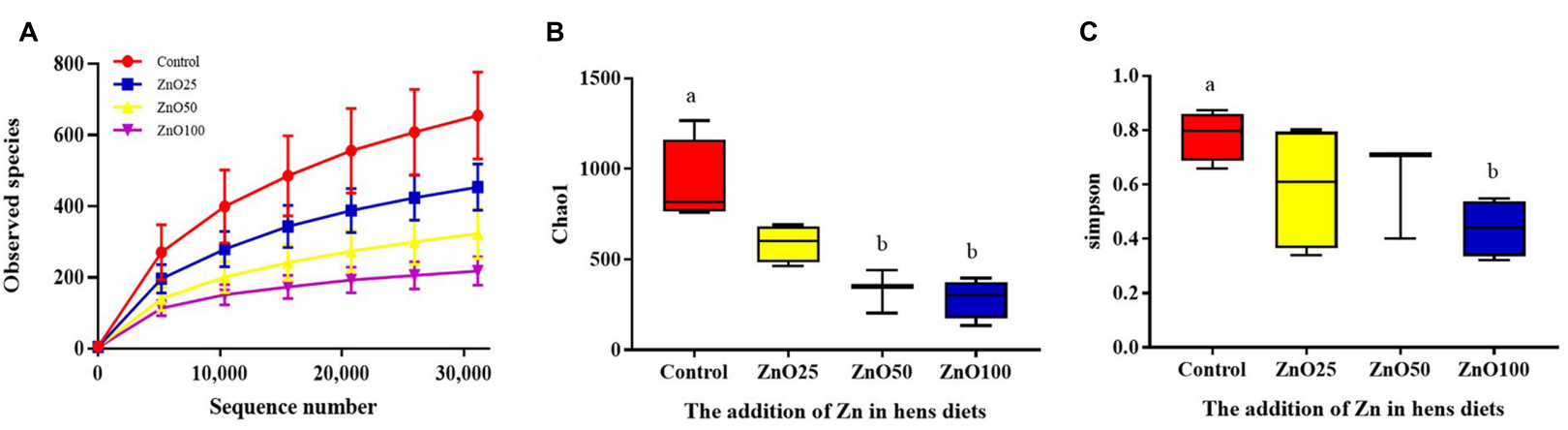

FIGURE 3 | The richness and the diversity of the bacterial community. The alpha index of the ileal microbiota: (A) Rarefaction curve, (B) Chao1 index, (C) Simpson index. 


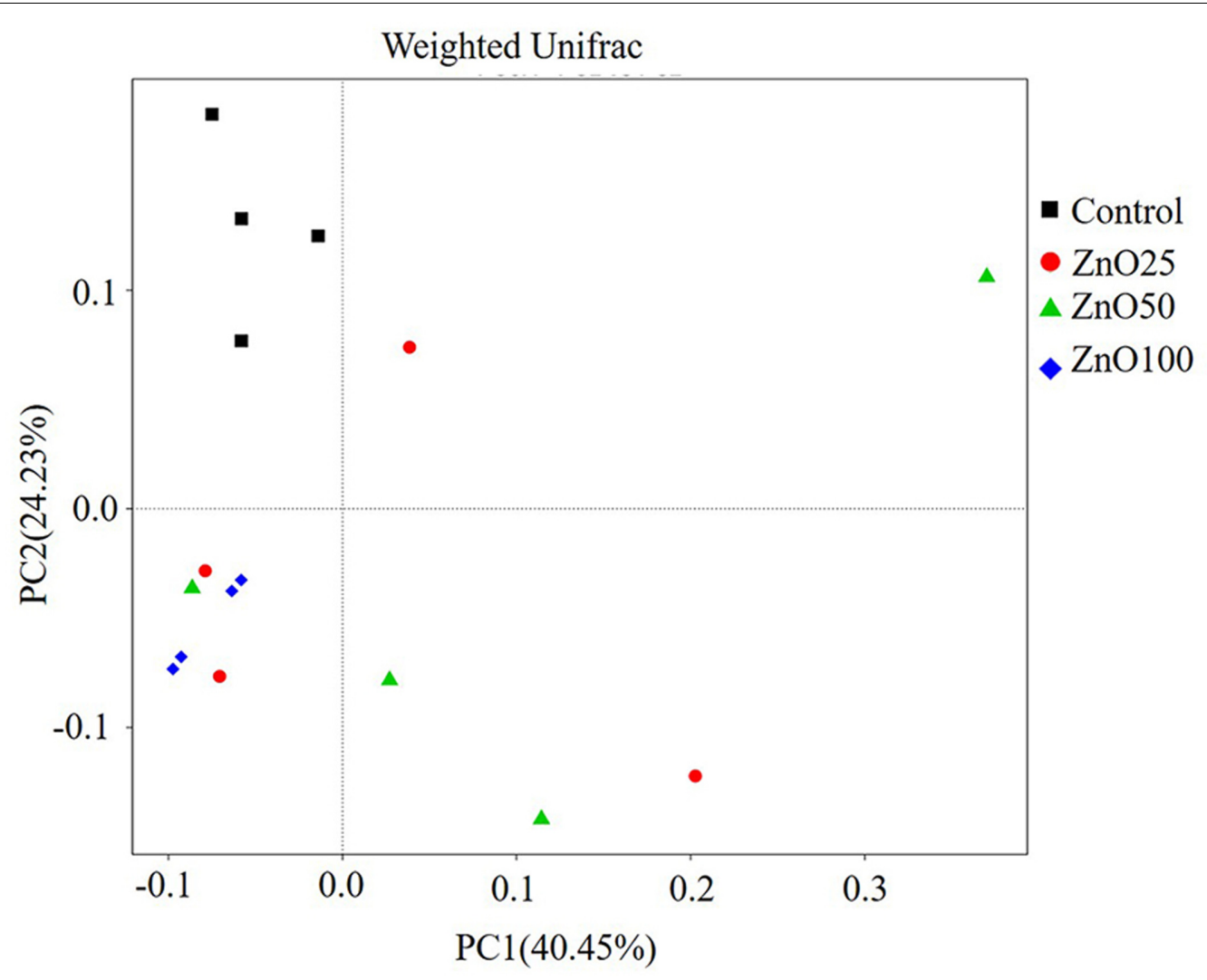

FIGURE 4 | The PCoA of the microflora in different treatments. After sequencing, Unifrac distances were calculated by QIIME (Version 1.7.0) and the PCoA was performed with R software (Version 2.15.3).
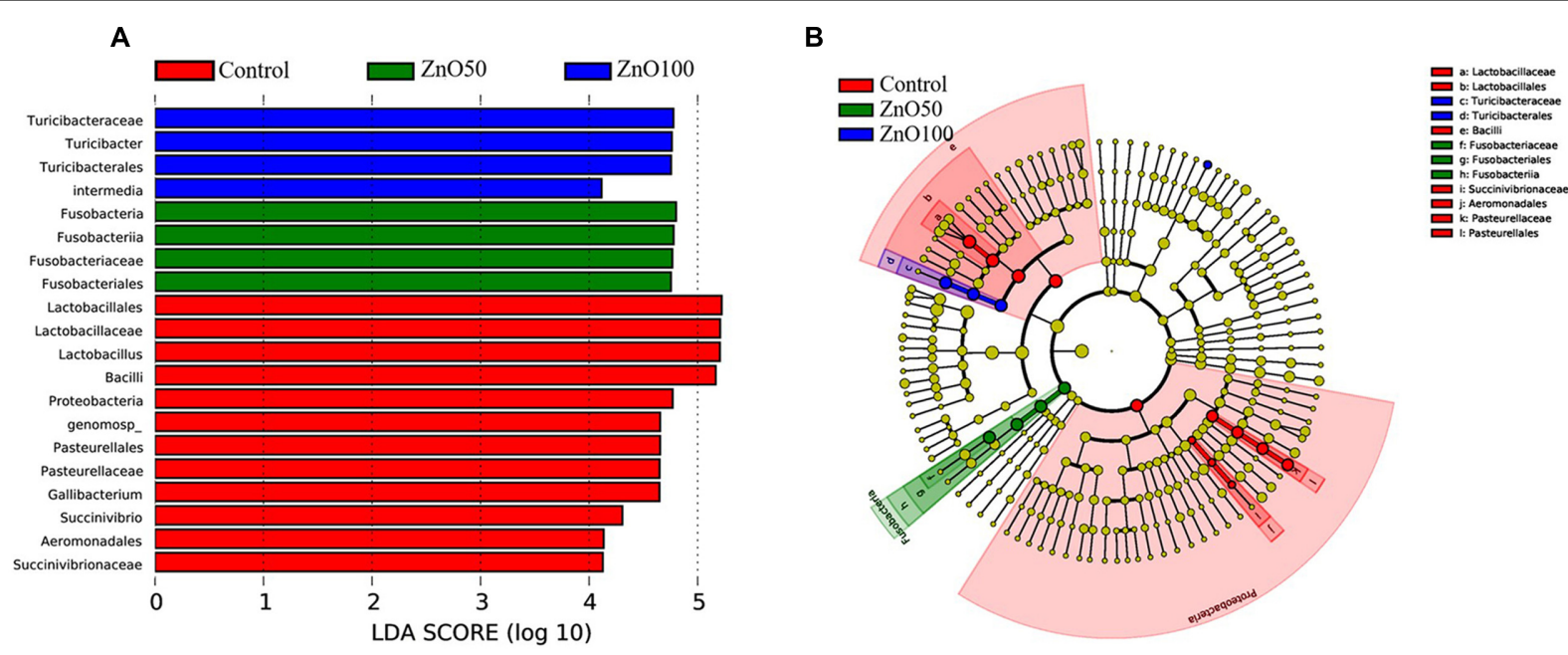

FIGURE 5 | Differences of bacterial abundance: (A) LDA distribution, (B) Cladogram. Linear discriminate analysis effect size (LEfSe) was performed to determine the difference in abundance; the threshold of LDA score was 4.0.

were negatively correlated with increased levels of $\mathrm{ZnO}$ NPs $(R=-0.636, P<0.01, n=16)$. Based on the alpha diversity index, richness (Chao1) and diversity (Simpson) were identified. Compared with the control group, richness was significantly decreased in the $\mathrm{ZnO}-\mathrm{NP}-50$ and $\mathrm{ZnO}-\mathrm{NP}-100$ treatments (Figure 3B), however, diversity was decreased only in treatment ZnO-NP-100 (Figure 3C). Moreover, based on the weighted unifrac distance, the PCoA visually showed that all ZnO NPs 
A

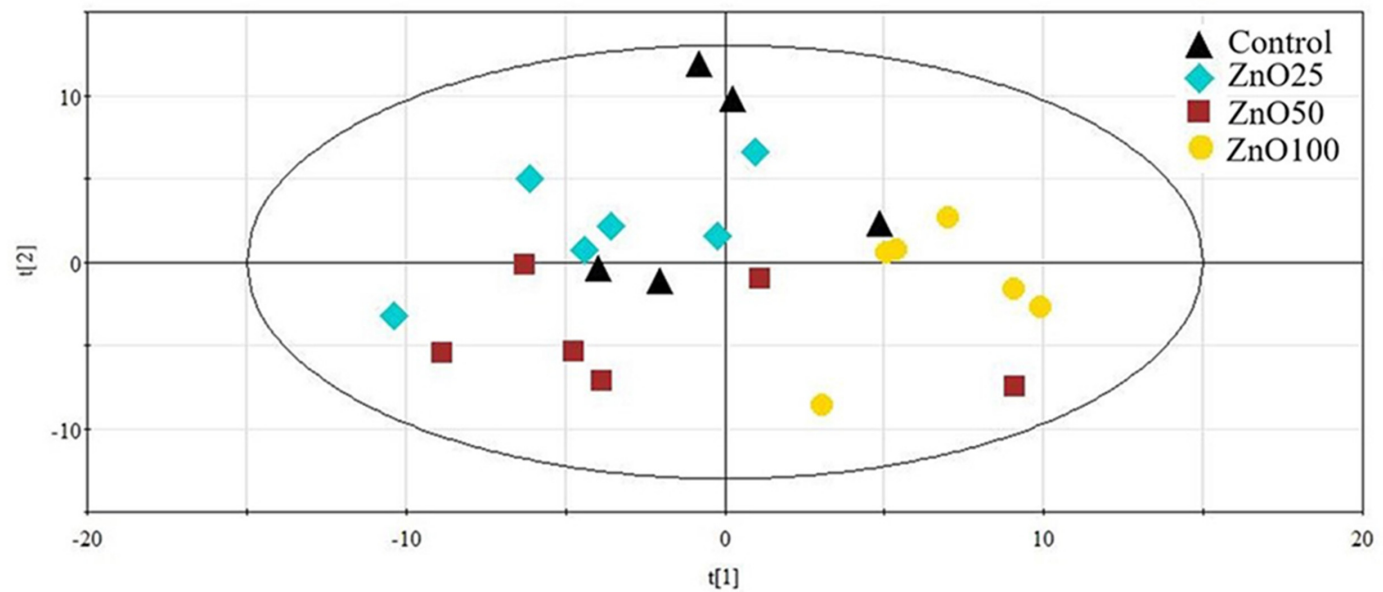

B
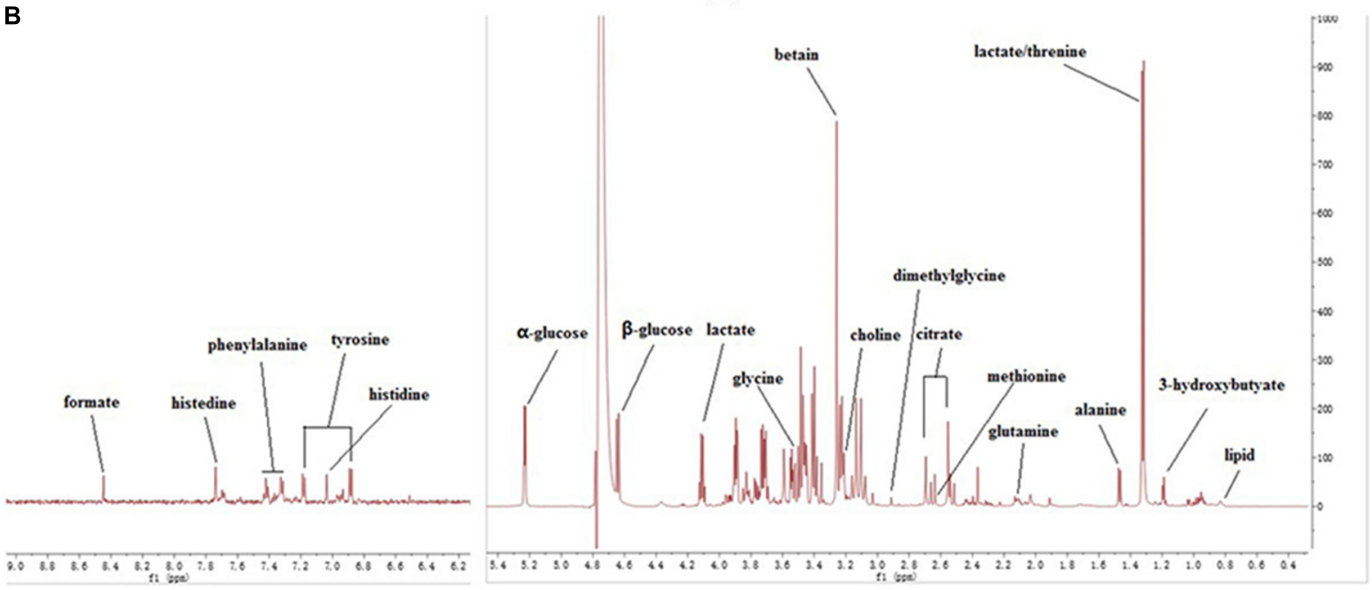

FIGURE 6 | Metabolites in blood plasma: (A) the PCA of metabolites, (B) Typical $600 \mathrm{MHz}{ }^{1} \mathrm{H}$ NMR spectra of plasma.

treatments were spatially separated from the control group, and Zn100 was the most distinctively different because of the low variability, and the explanation of PC1 and PC2 was $>60 \%$ (Figure 4).

\section{Characterization of Bacteria in Ileal Diagesta}

To evaluate the difference in bacterial content between the four groups, LEFSe were performed with an LDA value of four. There were 12 taxa that were significantly different in the control group, $\mathrm{ZnO}-\mathrm{NP}-50$, and ZnO-NP-100. When compared with $\mathrm{ZnO} \mathrm{NP}$ treatments, the abundance of Lactobacillales, Aeromonadales, and Pasteurellales were higher, and the Fusobacteriales and Turicibacterales were lower in the control group (Figure 5).

\section{Changes of Metabolites in Different Treatments}

Lipids, glucose, choline, lactate, citrate, and amino acids were identified from the ${ }^{1} \mathrm{H}$ NMR spectra. Data distribution was exhibit by PCA, and indicated that the ZnO-NP-100 treatment deviated from other groups (Figure 6). Metabolite analysis showed that levels of glucose, choline, lactate, citrate, glutamine, glycine, methionine, and tyrosine were altered by $\mathrm{ZnO} \mathrm{NP}$ administration (Figure 7).

\section{Correlations between Bacterial Richness and Metabolites in Plasma}

Since gut bacteria play a key role in food digestion, correlations between bacterial richness and metabolites were analyzed. The results showed that ileal digesta bacteria were positively related to choline, lactate, and methionine (Table 2).

\section{DISCUSSION}

Zinc oxide nanoparticles under $100 \mathrm{~nm}$ in diameter have special bioactivity and are widely used in many aspects of our lives. Since their first use, the adverse effects of $\mathrm{ZnO}$ NPs have been of great concern and have been widely studied in cells, animals, and even human subjects (Taccola et al., 2011; Gao et al., 2016; Han et al., 2016). However, the effects of ZnO NPs on gut microflora and metabolites in domestic animals had not been studied to-date. In this investigation, pullets were used to investigate the effects of $\sim 30 \mathrm{~nm}$ ZnO NPs on the gut microflora and blood metabolites 

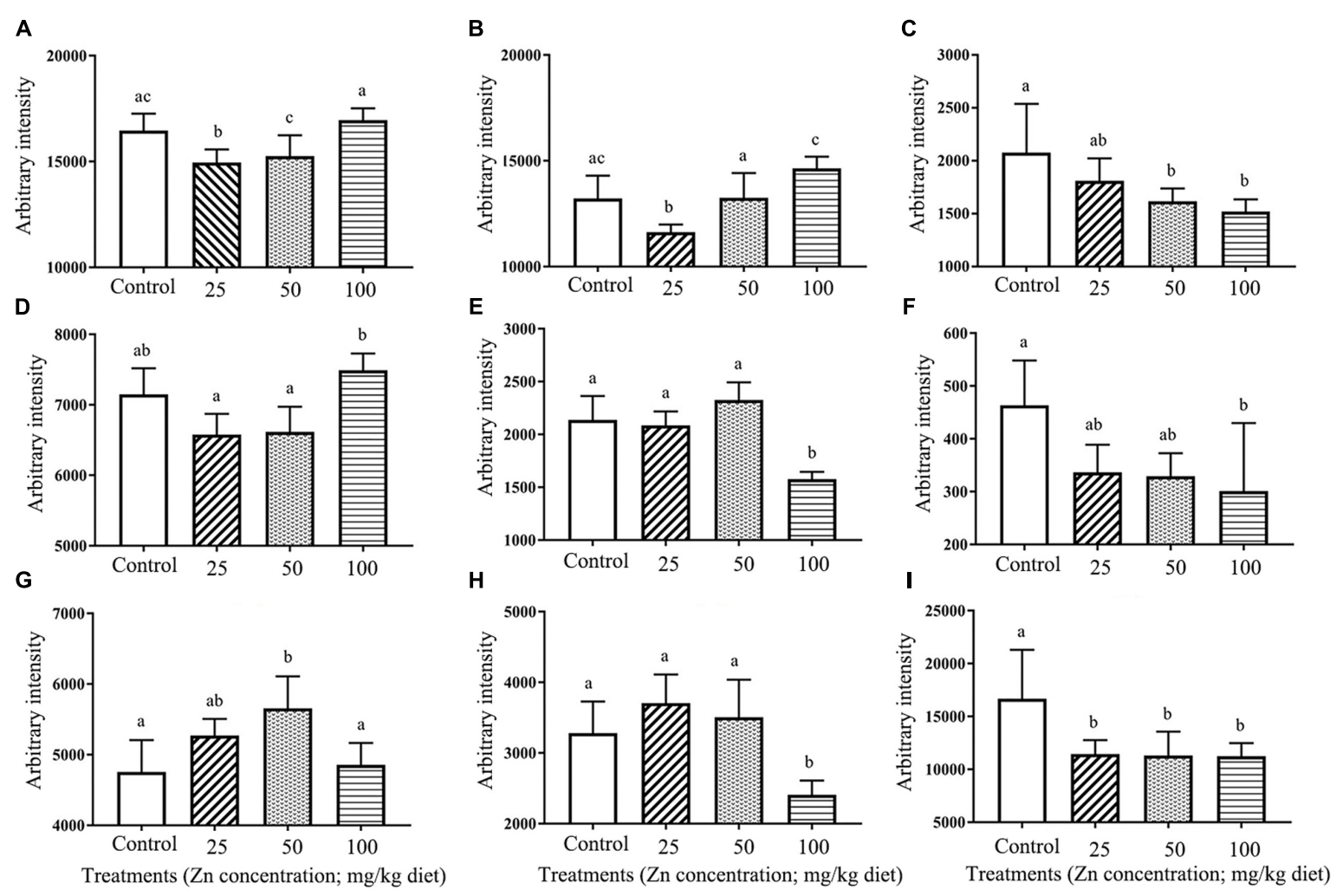

FIGURE 7 | Change in metabolites under different treatments: (A) $\alpha$-glucose, (B) $\beta$-glucose, (C) Choline, (D) Glycine, (E) Methionine, (F) Tyrosine, (G) Glutamine, (H) Citrate, (I) Lactate.

The dose dependent influence of $\mathrm{ZnO}$ NPs was investigated by different dose of Zinc addition around $45 \mathrm{ppm}$ (the recommend zinc dose for pullet).

\section{ZnO NPs Show Varying Effects on the Body Weight of Different Animal Species}

Fathi et al. (2016) first added different amounts of 35-45 nm ZnO NPs (10 mg/kg, $20 \mathrm{mg} / \mathrm{kg}$, and $40 \mathrm{mg} / \mathrm{kg}$ ) to diets and noted that this could improve the bodyweight of broiler chickens. Results in the current research revealed that the body weight of young hens remained unchanged by the addition of ZnO NPs, which indicates that different animal species and different animal life spans might respond differently to $\mathrm{ZnO}$ NPs. Previously, we discovered that $\mathrm{ZnO}$ NPs could decrease yolk lipids content (Zhao et al., 2016). Moreover, as gut microflora are involved in the metabolism (Nicholson et al., 2012), the pullet were selected as a model to verify the correlations between gut microflora and metabolites in this study.

\section{ZnO NP Effect on Gut Microbiota Varied According to Concentration, Particle Size, and Animal Species}

It is well known that $\mathrm{ZnO}$ NPs are good candidates for antimicrobial treatments for both $\mathrm{G}^{+}$and $\mathrm{G}^{-}$bacteria (Azam et al., 2012). They effectively inhibit pathogens such as Staphylococcus aureus (Geilich and Webster, 2013), Campylobacter jejuni (Xie et al., 2011), Escherichia coli O157:H7 (Liu et al., 2009), Listeria monocytogenes, and Salmonella Enteritidis (Jin et al., 2009). To date, the influence of ZnO NPs on the ecology of bacteria in domestic animals has been little

TABLE 2 | Correlations between the observed species and the major metabolites.

\begin{tabular}{|c|c|c|c|c|c|c|c|c|c|c|c|}
\hline & & Choline & Lactate & $\alpha$-glucose & $\beta$-glucose & Glutamine & Lipid & Tyrosine & Glycine & Methionine & Citrate \\
\hline \multirow[t]{3}{*}{$\begin{array}{l}\text { Observed } \\
\text { species }\end{array}$} & $\begin{array}{l}\text { Pearson } \\
\text { correlation }\end{array}$ & $0.769 * *$ & $0.509 *$ & 0.056 & -0.190 & -0.086 & -0.385 & 0.304 & 0.243 & $0.590 *$ & 0.229 \\
\hline & $\begin{array}{l}\text { Significance } \\
\text { (2-tailed) }\end{array}$ & 0.001 & 0.044 & 0.836 & 0.481 & 0.753 & 0.141 & 0.252 & 0.364 & 0.034 & 0.393 \\
\hline & $N$ & 16 & 16 & 16 & 16 & 16 & 16 & 16 & 16 & 13 & 16 \\
\hline
\end{tabular}

$* p<0.05 ; * * p<0.01$. 
understood. In the current study, we assessed changes in bacterial communities found within the ileal digesta of young hens, and suggest that the administration of $\mathrm{ZnO}$ NPs can decrease bacterial richness. Furthermore, the influence was enhanced by increased $\mathrm{ZnO} \mathrm{NP}$ concentration, and at the treatment level of $100 \mathrm{mg} / \mathrm{kg}$ it rearranged the predominant bacteria in ileal digesta.

The dominant bacteria in the intestines of animals and humans vary with organ, animal species and age. We found that Firmicutes $(>70 \%)$ and Proteobacteria (>5\%) were the dominant phyla of bacteria in the ileal digesta of all groups of young hens, and that ZnO NPs did not alter the relative abundance of Proteobacteria (Yausheva et al., 2016). In attempting to explain this disparity, firstly, Eisenia fetida are annelids, which are quite different to domestic animals. Secondly, bacteria throughout the entire intestinal tract were used for the latter authors' experiment, while we only focused on bacteria in ileal digesta. Finally, the size and amount of ZnO NPs used were different; the above-mentioned authors used $100 \mathrm{~nm}$ and $1000 \mathrm{mg} / \mathrm{kg}$, respectively, while $30 \mathrm{~nm}$ and 25, 50, and $100 \mathrm{mg} / \mathrm{kg}$ of $\mathrm{ZnO}$ NPs, respectively, were used in our investigation.

Reports have shown that Lactobacillus acidophilus (PTCC 1643) is inhibited by $1 \% \mathrm{ZnO}$ NPs in vitro (Kasraei et al., 2014). This is of concern because Lactobacillus is the predominant bacteria in animal and human ilea (Torok et al., 2011; Turroni et al., 2014; Jiao et al., 2016). In the current study, we also noted that the number and relative abundance of Lactobacillus in ileal digesta was remarkably reduced by $\mathrm{ZnO}$ NPs. The relative abundance of Lactobacillus in the control group was about $38.33 \%$; this agreed well with other studies and proved that Lactobacillus was the dominant bacteria in the ileum of young hens. As the dominant bacteria, it can be easily changed. It has been reported that Lactobacillus, especially Lactobacillus aviaries, is negatively correlated with the pathogen Clostridium perfringens in the ileum of broilers (Feng et al., 2010). Other authors found that a Lactobacillus population may be reduced through the feeding of antibiotic growth promoters (Lin et al., 2013). Lactobacillus aviaries and Lactobacillus salivarius are the predominant bacteria in the upper gastrointestinal tract of broilers (Gong et al., 2007). It has been confirmed that L. salivarius impedes broiler chicken performance by deconjugating bile salts (Harrow et al., 2007). On the other hand, our treatments produced no change in body weight compared with the control group. In our previous study, effects on egg laying frequency indicated that ZnO NPs decreased layer performance during the early period, while no difference was observed after 23 weeks of treatment (Zhao et al., 2016). It is therefore probable that $L$. salivarius is not sensitive to ZnO NPs.

Turicibacter is another dominant bacteria in the small intestine (Kamada et al., 2013), and its level could be increased by feeding an iron sulfate free diet (Werner et al., 2011). In the current study, ZnO NPs increased the relative abundance of Turicibacter in the $100 \mathrm{mg} / \mathrm{kg} \mathrm{ZnO}$ NPs treatment. Interestingly, when compared with the control group, the number of SMB53 was quite similar, while the relative abundance was increased in ZnO NPs treatments. The mechanism by which ZnO NPs affected Turicibacter and SMB53 was unclear. Turicibacter is considered as harmful bacteria and it was increased by $100 \mathrm{mg} / \mathrm{kg} \mathrm{ZnO}$ NPs treatment. This might suggest that bacterial tolerance has developed, or antibacterial activity has priorities in the complex environment.

Briefly, ZnO NPs affect the bacterial communities of ileal digesta. However, the research and reports on this subject were quite limited. Determination of the key mechanism (zinc, nanoparticles or both of them) involved in the changing the microbiota require further study.

\section{Metabolites in Blood Plasma Were Changed by ZnO NPs}

Due to their small size, $\mathrm{ZnO}$ NPs are able to enter cells and influence cell functions, including metabolism. Lai et al. (2015) discovered that $\mathrm{ZnO}$ NPs (mean diameter $63.1 \mathrm{~nm}$ ) located in lung cell mitochondria cause mitochondrial dysfunction and are a major source of ROS; in turn, the level of ROS affects energy metabolism. They also found that glucose consumption and lactate production were reduced at the concentration of $10 \mu \mathrm{g} / \mathrm{ml}$. Yan et al. (2012) found that rats given $50 \mathrm{~nm} \mathrm{ZnO}$ NPs $(100,300$, and $1000 \mathrm{mg} / \mathrm{kg}$ ) developed nephrotoxicity; the NPs changed kidney metabolism with a resulting increase in lactate and alpha-glucose and reduction in lipids and citrate in urine. The results of our current research did not all agree with earlier studies. We investigated blood plasma metabolites and the results showed that low levels of ZnO NPs $(25 \mathrm{mg} / \mathrm{kg})$ decreased glucose, while glucose (alpha and beta) was increased with increasing concentration of $\mathrm{ZnO}$ NPs and the level of beta-glucose was even higher than in the control. It is promising that $\mathrm{ZnO}$ NPs could decrease blood glucose at certain concentrations. Furthermore, we found that citrate was decreased at $100 \mathrm{mg} / \mathrm{kg} \mathrm{ZnO} \mathrm{NPs;} \mathrm{however,} \mathrm{lactate} \mathrm{and} \mathrm{choline} \mathrm{were}$ statistically significantly reduced by $\mathrm{ZnO}$ NPs, and lipids were also changed in our study; these results did not agree; with the findings of Yan et al. (2012). Basically, the animals and the samples used in the studies were different. Furthermore the above mentioned authors examined metabolism following the addition of $1000 \mathrm{mg} / \mathrm{kg} \mathrm{ZnO} \mathrm{NPs,} \mathrm{that} \mathrm{was} 10$-fold greater than our highest dosage. Although some differences were observed, it is certain that $\mathrm{ZnO}$ NPs can change metabolites by altering cell function.

\section{Metabolites Were Correlated with the Observed Species of Gut Microflora}

Gut microbiota are associated with development, disease, and metabolism. They are involved in the metabolism of shortchain fatty acids (SFCAs), glucose, lipids, vitamins, and choline etc., which are essential substrates for body maintenance and production (Nicholson et al., 2012). In the current research, the PCoA on bacteria template and the PCA on plasma showed that the $100 \mathrm{mg} / \mathrm{kg} \mathrm{ZnO}$ NPs treatment had significantly different results to the control group; this strongly suggests a connection between the gut bacterial community and metabolism. Nicholson et al. (2012) also reported that Lactobacillus is involved in the metabolism of bile acids, lipids, glucose, and D-lactate etc. 
We observed that bacterial numbers in ileal digesta were positively correlated with choline, lactate, and methionine, while other metabolites were not.

In summary, $\mathrm{ZnO} \mathrm{NPs}$ are widely used, could decrease the richness of bacterial communities, and inhibit Lactobacillus content. Bacterial richness in the ileum was correlated with some metabolites, including choline, lactate, and methionine. Together, $\mathrm{ZnO}$ NPs might regulate metabolism directly in host cells and indirectly via changing gut microbiota.

\section{DATA ACCESS}

The raw sequencing data generated in this study has been uploaded to the NCBI SRA database with the accession number: PRJNA361394 (http://www.ncbi.nlm.nih.gov/bioproject/PRJNA 361394).

\section{AUTHOR CONTRIBUTIONS}

YF, LM, and YZ designed and wrote the manuscript. WZ, JL, HZ, and $\mathrm{MC}$ performed the experiments. $\mathrm{ZH}, \mathrm{LL}$, and WS provided the data analysis and paper writing.

\section{REFERENCES}

Azam, A., Ahmed, A. S., Oves, M., Khan, M. S., Habib, S. S., and Memic, A. (2012). Antimicrobial activity of metal oxide nanoparticles against Gram-positive and Gram-negative bacteria: a comparative study. Int. J. Nanomed. 7, 6003-6009. doi: 10.2147/IJN.S35347

Caporaso, J. G., Kuczynski, J., Stombaugh, J., Bittinger, K., Bushman, F. D., Costello, E. K., et al. (2010). QIIME allows analysis of high-throughput community sequencing data. Nat. Methods 7, 335-336. doi: 10.1038/ nmeth.f.303

Costa, M. C., Bessegatto, J. A., Alfieri, A. A., Weese, J. S., Filho, J. A., and Oba, A. (2017). Different antibiotic growth promoters induce specific changes in the cecal microbiota membership of broiler chicken. PLoS ONE 12:e0171642. doi: 10.1371/journal.pone.0171642

Crisol-Martinez, E., Stanley, D., Geier, M. S., Hughes, R. J., and Moore, R. J. (2017). Understanding the mechanisms of zinc bacitracin and avilamycin on animal production: linking gut microbiota and growth performance in chickens. Appl. Microbiol. Biotechnol. doi: 10.1007/s00253-017-81939 [Epub ahead of print].

Edgar, R. C., Haas, B. J., Clemente, J. C., Quince, C., and Knight, R. (2011). UCHIME improves sensitivity and speed of chimera detection. Bioinformatics 27, 2194-2200. doi: 10.1093/bioinformatics/btr381

Fathi, M., Haydari, M., and Tanha, T. (2016). Effects of zinc oxide nanoparticles on antioxidant status, serum enzymes activities, biochemical parameters and performance in broiler chickens. J. Livest. Sci. Technol. 4, 7-13.

Feng, Y., Gong, J., Yu, H., Jin, Y., Zhu, J., and Han, Y. (2010). Identification of changes in the composition of ileal bacterial microbiota of broiler chickens infected with Clostridium perfringens. Vet. Microbiol. 140, 116-121. doi: 10.1016/j.vetmic.2009.07.001

Frohlich, E. E., and Frohlich, E. (2016). Cytotoxicity of nanoparticles contained in food on intestinal cells and the gut microbiota. Int. J. Mol. Sci. 17:509. doi: 10.3390/ijms17040509

Gao, F., Ma, N., Zhou, H., Wang, Q., Zhang, H., Wang, P., et al. (2016). Zinc oxide nanoparticles-induced epigenetic change and G2/M arrest are associated with apoptosis in human epidermal keratinocytes. Int. J. Nanomed. 11, 3859-3874. doi: 10.2147/IJN.S107021

Ge, Y., Schimel, J. P., and Holden, P. A. (2011). Evidence for negative effects of TiO2 and $\mathrm{ZnO}$ nanoparticles on soil bacterial communities. Environ. Sci. Technol. 45, 1659-1664. doi: 10.1021/es103040t

\section{FUNDING}

This work was financially supported by the Joint Special Funding of Shandong Natural Science Foundation (ZR2014CL033) and Funding of Qingdao Agricultural University.

\section{ACKNOWLEDGMENTS}

We would like to thank the workers in Maochangyuan Co. (Qiangdao, China). We are also grateful for the support of the Priority Academic Talent Team Cultivation Program of Shandong Colleges and Universities.

\section{SUPPLEMENTARY MATERIAL}

The Supplementary Material for this article can be found online at: http://journal.frontiersin.org/article/10.3389/fmicb. 2017.00992/full\#supplementary-material

FIGURE S1 | Characteristics of ZnO NPs. (A) Photo of ZnO NPs. (B) TEM image of ZnO NPs. (C) TEM photo of ZnO NPs in ovary indicated by the white arrow. (D) EDS picture of ZnO NPs in ovary, where three Zn peaks have shown.

Geilich, B. M., and Webster, T. J. (2013). Reduced adhesion of Staphylococcus aureus to $\mathrm{ZnO} / \mathrm{PVC}$ nanocomposites. Int. J. Nanomed. 8, 1177-1184. doi: 10.2147/IJN.S42010

Gong, J., Si, W., Forster, R. J., Huang, R., Yu, H., Yin, Y., et al. (2007). 16S rRNA gene-based analysis of mucosa-associated bacterial community and phylogeny in the chicken gastrointestinal tracts: from crops to ceca. FEMS Microbiol. Ecol. 59, 147-157. doi: 10.1111/j.1574-6941.2006.00193.x

Gong, J., Yu, H., Liu, T., Gill, J. J., Chambers, J. R., Wheatcroft, R., et al. (2008). Effects of zinc bacitracin, bird age and access to range on bacterial microbiota in the ileum and caeca of broiler chickens. J. Appl. Microbiol. 104, 1372-1382. doi: 10.1111/j.1365-2672.2007.03699.x

Guyard-Nicodeme, M., Keita, A., Quesne, S., Amelot, M., Poezevara, T., Le Berre, B., et al. (2016). Efficacy of feed additives against Campylobacter in live broilers during the entire rearing period. Poultt. Sci. 95, 298-305. doi: 10.3382/ ps/pev303

Haas, B. J., Gevers, D., Earl, A. M., Feldgarden, M., Ward, D. V., Giannoukos, G., et al. (2011). Chimeric 16S rRNA sequence formation and detection in Sanger and 454-pyrosequenced PCR amplicons. Genome Res. 21, 494-504. doi: 10.1101/gr.112730.110

Han, Z., Yan, Q., Ge, W., Liu, Z. G., Gurunathan, S., De Felici, M., et al. (2016). Cytotoxic effects of $\mathrm{ZnO}$ nanoparticles on mouse testicular cells. Int. J. Nanomed. 11, 5187-5203. doi: 10.2147/IJN. S111447

Harrow, S. A., Ravindran, V., Butler, R. C., Marshall, J. W., and Tannock, G. W. (2007). Real-time quantitative PCR measurement of ileal Lactobacillus salivarius populations from broiler chickens to determine the influence of farming practices. Appl. Environ. Microbiol. 73, 7123-7127.

Hu, Z., Lu, X., Sun, P., Hu, Z., Wang, R., Lou, C., et al. (2017). Understanding the performance of microbial community induced by $\mathrm{ZnO}$ nanoparticles in enhanced biological phosphorus removal system and its recoverability. Bioresour. Technol. 225, 279-285. doi: 10.1016/j.biortech.2016. 11.080

Jiao, J., Wu, J., Zhou, C., Tang, S., Wang, M., and Tan, Z. (2016). Composition of ileal bacterial community in grazing goats varies across non-rumination, transition and rumination stages of life. Front. Microbiol. 7:1364. doi: 10.3389/ fmicb.2016.01364

Jin, T., Sun, D., Su, J. Y., Zhang, H., and Sue, H. J. (2009). Antimicrobial efficacy of zinc oxide quantum dots against Listeria monocytogenes, Salmonella enteritidis, 
and Escherichia coli O157:H7. J. Food Sci. 74, M46-M52. doi: 10.1111/j.17503841.2008.01013.x

Kamada, N., Chen, G. Y., Inohara, N., and Nunez, G. (2013). Control of pathogens and pathobionts by the gut microbiota. Nat. Immunol. 14, 685-690. doi: $10.1038 /$ ni.2608

Kasraei, S., Sami, L., Hendi, S., Alikhani, M., Rezaei-Soufi, L., and Khamverdi, Z. (2014). Antibacterial properties of composite resins incorporating silver and zinc oxide nanoparticles on Streptococcus mutans and Lactobacillus. Restor. Dent. Endod. 39, 109-114. doi: 10.5395/rde.2014.39.2.109

Kim, M. H., Seo, J. H., Kim, H. M., and Jeong, H. J. (2014). Zinc oxide nanoparticles, a novel candidate for the treatment of allergic inflammatory diseases. Eur. J. Pharmacol. 738, 31-39. doi: 10.1016/j.ejphar.2014.05.030

Lai, X., Wei, Y., Zhao, H., Chen, S., Bu, X., Lu, F., et al. (2015). The effect of Fe2O3 and $\mathrm{ZnO}$ nanoparticles on cytotoxicity and glucose metabolism in lung epithelial cells. J. Appl. Toxicol. 35, 651-664. doi: 10.1002/jat.3128

Lin, J., Hunkapiller, A. A., Layton, A. C., Chang, Y. J., and Robbins, K. R. (2013). Response of intestinal microbiota to antibiotic growth promoters in chickens. Foodborne Pathog. Dis. 10, 331-337. doi: 10.1089/fpd.2012.1348

Lipovsky, A., Nitzan, Y., Gedanken, A., and Lubart, R. (2011). Antifungal activity of $\mathrm{ZnO}$ nanoparticles-the role of ROS mediated cell injury. Nanotechnology 22:105101. doi: 10.1088/0957-4484/22/10/105101

Liu, Y., He, L., Mustapha, A., Li, H., Hu, Z. Q., and Lin, M. (2009). Antibacterial activities of zinc oxide nanoparticles against Escherichia coli O157:H7. J. Appl. Microbiol. 107, 1193-1201. doi: 10.1111/j.1365-2672.2009.04303.x

Magoc, T., and Salzberg, S. L. (2011). FLASH: fast length adjustment of short reads to improve genome assemblies. Bioinformatics 27, 2957-2963. doi: 10.1093/ bioinformatics/btr507

Mercier-Bonin, M., Despax, B., Raynaud, P., Houdeau, E., and Thomas, M. (2016). Mucus and microbiota as emerging players in gut nanotoxicology: the example of dietary silver and titanium dioxide nanoparticles. Crit. Rev. Food Sci. Nutr. doi: 10.1080/10408398.2016.1243088 [Epub ahead of print].

Murugesan, G. R., Syed, B., Haldar, S., and Pender, C. (2015). Phytogenic feed additives as an alternative to antibiotic growth promoters in broiler chickens. Front. Vet. Sci. 2:21. doi: 10.3389/fvets.2015.00021

Nicholson, J. K., Holmes, E., Kinross, J., Burcelin, R., Gibson, G., Jia, W., et al. (2012). Host-gut microbiota metabolic interactions. Science 336, 1262-1267. doi: 10.1126/science. 1223813

Pan, D., and Yu, Z. (2014). Intestinal microbiome of poultry and its interaction with host and diet. Gut Microbes 5, 108-119. doi: 10.4161/gmic. 26945

Patel, P., Kansara, K., Senapati, V. A., Shanker, R., Dhawan, A., and Kumar, A. (2016). Cell cycle dependent cellular uptake of zinc oxide nanoparticles in human epidermal cells. Mutagenesis 31, 481-490. doi: 10.1093/mutage/ gew014

Pietroiusti, A., Magrini, A., and Campagnolo, L. (2016). New frontiers in nanotoxicology: gut microbiota/microbiome-mediated effects of engineered nanomaterials. Toxicol. Appl. Pharmacol. 299, 90-95. doi: 10.1016/j.taap.2015. 12.017

Reed, S., Neuman, H., Moscovich, S., Glahn, R. P., Koren, O., and Tako, E. (2015). Chronic zinc deficiency alters chick gut microbiota composition and function. Nutrients 7, 9768-9784. doi: 10.3390/nu7125497

Rodriguez, M. L., Rebole, A., Velasco, S., Ortiz, L. T., Trevino, J., and Alzueta, C. (2012). Wheat- and barley-based diets with or without additives influence broiler chicken performance, nutrient digestibility and intestinal microflora. J. Sci. Food Agric. 92, 184-190. doi: 10.1002/jsfa.4561

Ruiz, R., Peinado, M. J., Aranda-Olmedo, I., Abecia, L., Suarez-Pereira, E., Ortiz Mellet, C., et al. (2015). Effects of feed additives on ileal mucosa-associated microbiota composition of broiler chickens. J. Anim. Sci. 93, 3410-3420. doi: $10.2527 /$ jas.2015-8905

Santimano, M. C., and Kowshik, M. (2013). Altered growth and enzyme expression profile of $\mathrm{ZnO}$ nanoparticles exposed non-target environmentally beneficial bacteria. Environ. Monit. Assess. 185, 7205-7214. doi: 10.1007/s10661-01330946

Saptarshi, S. R., Duschl, A., and Lopata, A. L. (2015). Biological reactivity of zinc oxide nanoparticles with mammalian test systems: an overview. Nanomedicine (Lond.) 10, 2075-2092. doi: 10.2217/nnm.15.44

Shao, Y., Lei, Z., Yuan, J., Yang, Y., Guo, Y., and Zhang, B. (2014). Effect of zinc on growth performance, gut morphometry, and cecal microbial community in broilers challenged with Salmonella enterica serovar typhimurium. J. Microbiol. 52, 1002-1011. doi: 10.1007/s12275-014-4347-y

Shin, S. C., Kim, S. H., You, H., Kim, B., Kim, A. C., Lee, K. A., et al. (2011). Drosophila microbiome modulates host developmental and metabolic homeostasis via insulin signaling. Science 334, 670-674. doi: 10.1126/science. 1212782

Silva, L. R., and Girard, D. (2016). Human eosinophils are direct targets to nanoparticles: zinc oxide nanoparticles $(\mathrm{ZnO})$ delay apoptosis and increase the production of the pro-inflammatory cytokines IL-1beta and IL-8. Toxicol. Lett. 259, 11-20. doi: 10.1016/j.toxlet.2016.07.020

Sirelkhatim, A., Mahmud, S., Seeni, A., Kaus, N. H. M., Ann, L. C., Bakhori, S. K. M., et al. (2015). Review on zinc oxide nanoparticles: antibacterial activity and toxicity mechanism. Nano-Micro Lett. 7, 219-242. doi: 10.1007/s40820015-0040-x

Taccola, L., Raffa, V., Riggio, C., Vittorio, O., Iorio, M. C., Vanacore, R., et al. (2011). Zinc oxide nanoparticles as selective killers of proliferating cells. Int. J. Nanomed. 6, 1129-1140. doi: 10.2147/IJN.S16581

Taylor, A. A., Marcus, I. M., Guysi, R. L., and Walker, S. L. (2015). Metal oxide nanoparticles induce minimal phenotypic changes in a model colon gut microbiota. Environ. Eng. Sci. 32, 602-612.

Torok, V. A., Allison, G. E., Percy, N. J., Ophel-Keller, K., and Hughes, R. J. (2011). Influence of antimicrobial feed additives on broiler commensal posthatch gut microbiota development and performance. Appl. Environ. Microbiol. 77, 3380-3390. doi: 10.1128/AEM.0230010

Turroni, F., Ventura, M., Butto, L. F., Duranti, S., O’Toole, P. W., Motherway, M. O., et al. (2014). Molecular dialogue between the human gut microbiota and the host: a Lactobacillus and Bifidobacterium perspective. Cell Mol. Life. Sci. 71, 183-203. doi: 10.1007/s00018-013-13180

Wan, Q., He, Q., Deng, X., Hao, F., Tang, H., and Wang, Y. (2016). Systemic metabolic responses of broiler chickens and piglets to acute T-2 toxin intravenous exposure. J. Agric. Food Chem. 64, 714-723. doi: 10.1021/acs.jafc. $5 \mathrm{~b} 05076$

Wang, B., Zhang, Y., Mao, Z., Yu, D., and Gao, C. (2014). Toxicity of ZnO nanoparticles to macrophages due to cell uptake and intracellular release of zinc ions. J. Nanosci. Nanotechnol. 14, 5688-5696.

Werner, T., Wagner, S. J., Martinez, I., Walter, J., Chang, J. S., Clavel, T., et al. (2011). Depletion of luminal iron alters the gut microbiota and prevents Crohn's disease-like ileitis. Gut 60, 325-333. doi: 10.1136/gut.2010.216929

Xie, Y., He, Y., Irwin, P. L., Jin, T., and Shi, X. (2011). Antibacterial activity and mechanism of action of zinc oxide nanoparticles against Campylobacter jejuni. Appl. Environ. Microbiol. 77, 2325-2331. doi: 10.1128/AEM.0214910

Yan, G., Huang, Y., Bu, Q., Lv, L., Deng, P., Zhou, J., et al. (2012). Zinc oxide nanoparticles cause nephrotoxicity and kidney metabolism alterations in rats. J. Environ. Sci. Health Tox Hazard. Subst. Environ. Eng. 47, 577-588. doi: $10.1080 / 10934529.2012 .650576$

Yausheva, E., Sizova, E., Lebedev, S., Skalny, A., Miroshnikov, S., Plotnikov, A., et al. (2016). Influence of zinc nanoparticles on survival of worms Eisenia fetida and taxonomic diversity of the gut microflora. Environ. Sci. Pollut. Res. Int. 23, 13245-13254. doi: 10.1007/s11356-016-6474-y

Yu, Y., Lee, C., Kim, J., and Hwang, S. (2005). Group-specific primer and probe sets to detect methanogenic communities using quantitative real-time polymerase chain reaction. Biotechnol. Bioeng. 89, 670-679. doi: 10.1002/bit. 20347

Zhao, Y., Li, L., Zhang, P. F., Liu, X. Q., Zhang, W. D., Ding, Z. P., et al. (2016). Regulation of egg quality and lipids metabolism by Zinc Oxide Nanoparticles. Poultt. Sci. 95, 920-933. doi: 10.3382/ps/pev436

Conflict of Interest Statement: The authors declare that the research was conducted in the absence of any commercial or financial relationships that could be construed as a potential conflict of interest.

Copyright () 2017 Feng, Min, Zhang, Liu, Hou, Chu, Li, Shen, Zhao and Zhang. This is an open-access article distributed under the terms of the Creative Commons Attribution License (CC BY). The use, distribution or reproduction in other forums is permitted, provided the original author(s) or licensor are credited and that the original publication in this journal is cited, in accordance with accepted academic practice. No use, distribution or reproduction is permitted which does not comply with these terms. 